\title{
Myocardial blood flow quantitation with the SPECT technique: Is it ready to be a substitute for PET myocardial blood flow quantitation?
}

\author{
Wei Fang, $\mathrm{MD} / \mathrm{PhD}$, ${ }^{\mathrm{a}}$ and Bailing $\mathrm{Hsu}, \mathrm{PhD}^{\mathrm{b}}$ \\ a Department of Nuclear Medicine, Fuwai Hospital, National Center for Cardiovascular Diseases, \\ Chinese Academy of Medical Sciences and Peking Union Medical College, Beijing, China \\ b Nuclear Science and Engineering Institute, University of Missouri-Columbia, Columbia, MO
}

Received May 30, 2021; accepted Jun 1, 2021

doi: $10.1007 / \mathrm{s} 12350-021-02697-5$

See related article, pp. 3137-3151

In the last ten years, myocardial blood flow quantitation (MBF) with the SPECT technique has been continuously investigated for its capability toward the clinical utilization. The outcome is to establish a widely available clinical tool that can offer an accurate measurement of MBF close to PET MBF quantitation for the diagnosis and prognosis of coronary artery disease (CAD). This clinical solution is particularly attractive and demanded for area (e.g., Asia and Australia) where PET MBF quantitation is not applicable due to the lack of suitable PET myocardial flow tracers as the main barrier. Even in areas (e.g., North America and Europe) where PET MBF quantitation is available, SPECT MBF quantitation still draws large interest because of its simplicity and lower cost to integrate into the SPECT user environment.

The fundamental requisite for SPECT MBF quantitation depends on three technical components: fast dynamic SPECT acquisition, suitable SPECT myocardial flow tracers, and dedicated software. Nowadays, SPECT myocardial flow tracers $\left({ }^{99 \mathrm{~m}} \mathrm{Tc}-\right.$ Sestamibi and ${ }^{99 m}$ Tc-Tetrofosmin) and dedicated software (4DM SPECT CFR, FlowQuant, QPET, and MyoFlowQ) are world-wide available. The remaining challenge comes

Reprint requests: Bailing $\mathrm{Hsu}, \mathrm{PhD}$, Nuclear Science and Engineering Institute, University of Missouri-Columbia, Columbia, MO; bailinghsu@gmail.com

J Nucl Cardiol 2022;29:3152-4.

1071-3581/\$34.00

Copyright (C) 2021 American Society of Nuclear Cardiology. from the requirement of SPECT cameras capable of performing fast dynamic SPECT acquisition in at least 10 -second time resolution to effectively image the rapid radiotracer distribution in myocardium. In fact, this fast dynamic SPECT acquisition mode is only applicable in certain SPECT camera models with $\mathrm{NaI}(\mathrm{Tl})$ detector and rapid rotating gantry (RRG) (e.g., Symbia SPECT and SPECT/CT family) $)^{1,2}$ or cadmium zinc telluride (CZT) detector and list-mode data acquisition (e.g., Discovery NM530c and D-SPECT),${ }^{3-5}$ leaving the technique of SPECT MBF quantitation not implementable for all SPECT cameras currently serving in clinics. However, the situation may gradually change with the new installation of SPECT and SPECT/CT cameras containing this important function.

In the review article composed by Zavadovsky and et al, the current status of CZT-SPECT myocardial blood flow and reserve assessment are comprehensively reviewed, including imaging protocols, image corrections, validations with PET quantitation, angiography and fraction flow reserve, and technical/clinical limitations. ${ }^{6}$ This review article provides the most updated information in many aspects for readers to fully learn about SPECT MBF quantitation and is highly recommended for the interested. Compared with RRG SPECT, the major advantages of CZT SPECT are recognized as higher count rate and energy resolution that can allow for using lower-dose injection and less scatter contamination for correction. Another advantage is marked as the stationary gantry design to avoid a correction for angular inconsistency as required for RRG SPECT. Due to small image field-of-view, the disadvantage comes from the unavoided low-dose pre-injection for myocardium localization. While not appropriately corrected, myocardial uptake from the pre-injected dose can 


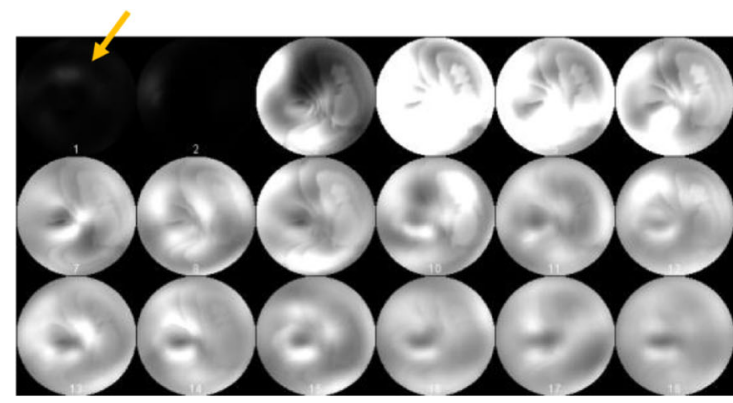

$\widehat{\text { Rest Dynamic Polar Maps }}$

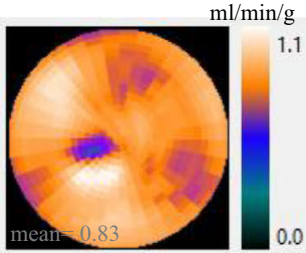

Uncorrected

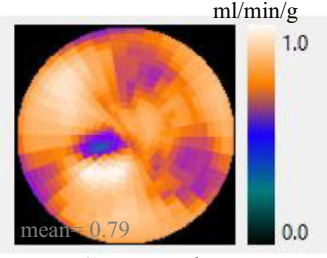

Corrected

Rest MBF

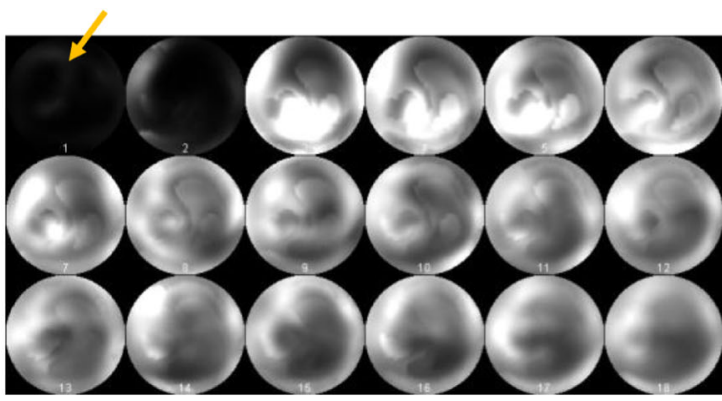

Stress Dynamic Polar Maps

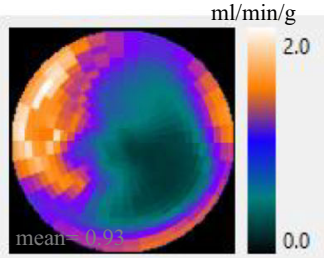

Uncorrected

Stress MBF

Figure 1. An example of CZT-SPECT MBF quantitation with and without the correction of lowdose pre-injection for myocardium localization. Arrows point to the first frame of dynamic polar maps having myocardial uptake due to the pre-injection.

introduce around $5 \%-20 \%$ bias to the flow data depending on the ratio of pre-injected and injected doses, rest/stress stages and individual patient (Figure 1). Nonetheless, RRG SPECT and CZT SPECT require full image corrections, including corrections for attenuation, scatter, spatial resolution (required for RRG), image noise, patient motion, tracer extraction, physiology variance in the rest, and the rest residual uptake in the stress data for the one-day protocol in order to perform accurate quantitation close to PET. ${ }^{7-10}$ Particularly, as attenuation correction is not applicable, the quantitation of rest and stress $\mathrm{MBF}$ in $(\mathrm{ml} / \mathrm{min} / \mathrm{g})$ will be few times overestimated as myocardial flow reserve can still be preserved. ${ }^{5,11}$ Lacking attenuation and associated corrections would further prevent the implementation of stress-only protocol in patients without a history of myocardial infarction like PET MBF quantitation, ${ }^{12,13}$ which is the key to effectively reduce the patient radiation exposure and increase the laboratory efficiency. It is important to point out that when necessary image corrections are applied, both RRG SPECT and CZT SPECT can consistently quantify very close MBF values on the same cohort. ${ }^{14}$ In the Zavadovsky's review article, the clinical validation of SPECT MBF quantitation vs PET was carefully reviewed. As the accuracy of SPECT MBF quantitation is warranted, its clinical strength should be recognized as the strength of PET MBF quantitation in the detection of multi-vessel CAD, functional significance in intermediate coronary lesion (40\%-90\% stenosis), early asymptomatic $\mathrm{CAD}, \quad$ and microvascular dysfunction. ${ }^{15-18}$

It is generally believed the important role of SPECT MBF quantitation as a substitute for PET MBF quantitation is going on its way when more and more clinical data are being published. This accomplishment is expected and will continuously facilitate the clinical utilization of SPECT MBF quantitation, especially for areas where PET MBF quantitation is not accessible.

\section{Disclosures}

Drs. Fang, Huang and Hsu have nothing to disclose.

\section{References}

1. Hsu B, Chen FC, Wu TC, Huang WS, Hou PN, Chen CC, Hung GU. Quantitation of myocardial blood flow and myocardial flow reserve with $99 \mathrm{mTc}$-sestamibi dynamic SPECT/CT to enhance detection of coronary artery disease. Eur J Nucl Med Mol Imagin 2014; 41:2294-06

2. Chen LC, Hung HF, Jong BH, Lin SC, Yeh CL, Ku CT et al. A method to measure the extent of myocardial ischemia and steal with SPECT myocardial blood flow quantitation. Ann Nucl Med 2020; 34:682-90

3. Ben Bouallègue $F$, Roubille $F$, Lattuca $B$ et al. SPECT myocardial perfusion reserve in patients with multivessel coronary disease: Correlation with angiographic findings and invasive fractional flow reserve measurements. J Nucl Med 2015; 56:1712-17

4. Shiraishi S, Tsuda N, Sakamoto F et al. Clinical usefulness of quantification of myocardial blood flow and flow reserve using 
CZT-SPECT for detecting coronary artery disease in patients with normal stress perfusion imaging. J Cardiol 2020; 75:400-09

5. Agostini D, Roule V, Nganoa $C$ et al. First validation of myocardial flow reserve assessed by dynamic $99 \mathrm{mTc}$-sestamibi CZT-SPECT camera: Head to head comparison with 15O-water PET and fractional flow reserve in patients with suspected coronary artery disease. The WATERDAY study. Eur J Nucl Med Mol Imaging 2018; 45:1079-90

6. Zavadovsky KV, Mochula AV, Maltseva AN, Shipulin VV, Sazonova SI, Gulya MO, Liga R, Gimelli A. The current status of CZT SPECT myocardial blood flow and reserve assessment: Tips and tricks. J Nucl Cardiol. 2021. https://doi.org/10.1007/s12350021-02620-y

7. Hyafil F, Chequer R, Sorbets E et al. Head-to-head comparison of the diagnostic performances of Rubidium-PET and SPECT with CZT camera for the detection of myocardial ischemia in a population of women and overweight individuals. J Nucl Cardiol 2020; 27:755-68

8. Hsu B, Hu LH, Yang BH et al. SPECT myocardial blood flow quantitation toward clinical use: A comparative study with $13 \mathrm{~N}$ Ammonia PET myocardial blood flow quantitation. Eur J Nucl Med Mol Imaging 2017; 44:117-28

9. Wells RG, Marvin B, Poirier M, Renaud J, deKemp RA, Ruddy TD. Optimization of SPECT measurement of myocardial blood flow with corrections for attenuation, motion, and blood binding compared with PET. J Nucl Med 2017; 58:2013-19

10. Giubbini R, Bertoli M, Durmo R, Bonacina M, Peli A, Faggiano I et al. Comparison between N13NH3-PET and 99mTc-Tetrofosmin-CZT SPECT in the evaluation of absolute myocardial blood flow and flow reserve. J Nucl Cardiol. 2019. https://doi.org/10. 1007/s12350-019-01939-x

11. Wang L, Wu D, Yang Y, Chen IJ, Lin CY, Hsu B et al. Avoiding full corrections in dynamic SPECT images impacts the performance of SPECT myocardial blood flow quantitation. J Nucl Cardiol. Nucl Cardiol 2017; 24:1332-46
12. Danad I, Uusitalo V, Kero T et al. Quantitative assessment of myocardial perfusion in the detection of significant coronary artery disease: Cutoff values and diagnostic accuracy of quantitative [(15)O]H2O PET imaging. J Am Coll Cardiol 2014; 64:1464-75

13. Joutsiniemi E, Saraste A, Pietilä $M$ et al. Absolute flow or myocardial flow reserve for the detection of significant coronary artery disease? Eur Heart J Cardiovasc Imaging 2014; 15:659-65

14. Ma R, Wang L, Wu D, Wang M, Sun X, Hsu B, Fang W. Myocardial blood flow quantitation in patients with congestive heart failure: Head-to-head comparison between rapid-rotating gantry SPECT and CZT SPECT. J Nucl Cardiol 2020; 27:2287-02

15. Ziadi MC, Dekemp RA, Williams K, Guo A, Renaud JM, Chow $\mathrm{BJ}$ et al. Does quantification of myocardial flow reserve using rubidium- 82 positron emission tomography facilitate detection of multivessel coronary artery disease? J Nucl Cardiol 2012; 19:67080

16. Murthy VL, Bateman TM, Beanlands RS, Berman DS, BorgesNeto S, Chareonthaitawee P et al. Correction to: Clinical quantification of myocardial blood flow using PET: Joint Position Paper of the SNMMI Cardiovascular Council and the ASNC. J Nucl Cardiol 2018; 25:2189-90

17. Dayanikli F, Grambow D, Muzik O, Mosca L, Rubenfire M, Schwaiger M. Early detection of abnormal coronary flow reserve in asymptomatic men at high risk for coronary artery disease using positron emission tomography. Circulation 1994; 90:808-17

18. Graf S, Khorsand A, Gwechenberger M, Novotny C, Kletter K, Sochor $\mathrm{H}$ et al. Typical chest pain and normal coronary angiogram: cardiac risk factor analysis versus PET for detection of microvascular disease. J Nucl Med 2007; 48:175-81

Publisher's Note Springer Nature remains neutral with regard to jurisdictional claims in published maps and institutional affiliations. 